\title{
Herramientas de mediación didáctica en entornos virtuales: Las webqests.
}

\author{
Andrés García Manzano \\ agmemaptel .es \\ Profesor de Secundaria
}

\section{Introducción}

El fenómeno Internet no deja de crecer de manera imparable, convirtiéndose en fuente hegemónica de información y servicios digitales a escala mundial. Las relaciones interpersonales, los procesos laborales y, en general, los hábitos de no pocos ciudadanos se están viendo modificados de manera sustancial debido al advenimiento de la Cibersociedad. La actividad escolar no debe descolgarse de este proceso continuo de inmersión en la cultura digital, porque Internet puede constituir una eficiente herramienta para mejorar los procesos de enseñanza-aprendizaje.

Sin embargo, el recurso a la web como instrumento didáctico genera numerosos problemas que es necesario solventar si queremos que nuestros alumnos aprendan a emplear eficientemente este medio:

1. La formación en TIC de los docentes. Por lo general escasa o cuando menos heterogénea. Aún faltan programas para la formación del profesorado, no tanto por lo que respecta al uso puramente instrumental de los medios informáticos (para eso están las academias y los manuales técnicos) sino al desarrollo de estrategias didácticas que permitan aplicar estas herramientas de manera adecuada en entornos reales de aprendizaje.

2. Los conocimientos informáticos del alumnado son insuficientes, dispersos y pobres. La Informática sigue siendo una optativa y el número de horas semanales que los alumnos pasan al ordenador resulta escaso.

3. El acceso a Internet es problemático por lo que respecta a la calidad, disponibilidad, dispersión e intencionalidad de los contenidos. Un porcentaje insignificante de sitios web tiene carácter educativo, de ellos el $80 \%$ están en inglés. Por otro lado, los alumnos suelen nadar inermes en un océano de información confusa y fragmentaria, cuya calidad y fiabilidad escapa totalmente a su control.

4. Por último, está la cara oculta de Internet, su vertiente tortuosa, delictiva y perturbadora: Invitaciones al sexo fácil, a la violencia y al consumo irracional que en mentes aún inmaduras pueden ocasionar daños irreparables.

Debido a los problemas enumerados, queda claro que, en el uso educativo de este medio, los docentes no podemos improvisar. Urge el desarrollo de estrategias pedagógicas que vayan más allá de la mera exhibición de habilidades técnicas y se incorporen eficientemente a los diseños curriculares de las asignaturas, ciclos y niveles del sistema educativo.

En este contexto, la propuesta de Bernie Dodge de elaborar un protocolo estandarizado (webquest) para el aprovechamiento de Internet en tareas docentes resulta especialmente útil, tanto por el carácter abierto de la metodología -fácilmente aplicable a cualquier nivel y asignatura- como por la sencillez de los materiales que deberá generar el profesor, lo que pone esta metodología al alcance de un buen 
número de profesionales de la enseñanza sin conocimientos de diseño web.

Pero además, desde el punto de vista de la tecnología educativa, la webquest es un buen instrumento pedagógico porque se basa en una metodología centrada en procesos y objetivos, estructurada como modelo informal de investigación en la que el alumno interviene de manera activa y sistemática. De este modo, el recurso a las TIC se realiza de forma natural y diversificada, en situaciones reales de aprendizaje y en respuesta a cuestiones que el alumno -a menudo- deberá resolver mediante el trabajo colaborativo. Por lo general, forman grupos de trabajo en los que se asignan roles y se diversifican las tareas. A menudo, la interacción entre alumnos también está mediada por la comunicación on-line o requiere contactar por medios telemáticos, para resolver ciertas tareas con otos agentes externos al grupo.

Al basarse en una metodología de trabajo centrada en la realización de actividades que el alumno conoce de antemano, se está propiciando también cierta autonomía educativa (o autoaprendizaje). Si bien, el repertorio de tareas -y sitios web de visita obligada- establecidos por el profesor suele acotar bastante el espacio de trabajo, evitando una dispersión innecesaria y rentabilizando el tiempo dedicado a la consecución de los objetivos curriculares.

Así mismo, esta metodología supone un reto intelectual, estimulando, en el marco de una filosofía educativa constructivista, el desarrollo de numerosos procesos cognitivos que conducen, en última instancia, a transformar la información en conocimiento y el conocimiento en saber operativo.

En definitiva: Las webquest constituyen, hoy por hoy, una de las metodologías más consolidadas de aprendizaje colaborativo en el aula empleando Internet como recurso didáctico y como instrumento de mediación tecnológica.

\section{2. ¿Qué es una webquest?}

La webquest es un protocolo didáctico que pretende aprovechar el potencial de la Red como herramienta eficiente tanto en los procesos de búsqueda, valoración y análisis crítico de la información, como en las estrategias colaborativas implicadas en la construcción social del conocimiento. Bernie Dodge [1] (1995) las define como:

An inquiry-oriented activity in which most or all of the information used by learners is drawn from the Web. WebQuests are designed to use learners' time well, to focus on using information rather than looking for it, and to support learners' thinking at the levels of analysis, synthesis, and evaluation. [2]

Se trata, por tanto, de una "propuesta informal" de investigación dirigida al alumnado, en la que los recursos empleados provienen parcial o totalmente de Internet. Los alumnos, trabajando sobre una unidad didáctica diseñada por el profesor y concebida como página web, deberán actuar de manera colaborativa en la resolución de una serie de tareas que implican:

• Búsqueda, análisis y contrastación de la información recopilada.

- Organización y representación del conocimiento: Resúmenes, esquemas, diagramas, mapas conceptuales.

- Construcción social del conocimiento: Deliberación, asunción de roles, debate, puesta en común, puesta en práctica de estrategias discursivas y búsqueda de espacios para el consenso.

- Elaboración de conclusiones: De manera individual y en grupo.

- Valoración -y, a menudo, autoevaluación- del proceso de trabajo con la webwest y grado de consecución de los objetivos previstos.

De este modo la webquest busca producir aprendizajes significativos centrados en un determinado 
nivel, materia curricular o unidad didáctica, a la vez que propicia en el alumnado una nueva metodología de trabajo basada en el uso eficiente de las TIC en entornos educativos. Así, la adquisición de competencias informáticas por parte del alumno -y no pocas veces del propio profesoradquiere un valor pedagógico que refuerza de manera transversal los procesos de búsqueda, transformación y producción de conocimientos.

Por lo que se refiere a la metodología y partes en que se estructura una webquest, cabe decir que se trata de un protocolo estandarizado [3] de trabajo colaborativo que se compone de los siguientes elementos:

1. Introducción.- Donde se expone el tema central y el ámbito que abarca. Su objetivo es captar la atención del alumno y acotar con precisión la unidad didáctica, tema, subtema, apartado, contenido transversal, etc. que se va a trabajar.

2. Tarea.- Apartado en el que se especifica el tipo de actividades que realizarán los alumnos. Éstos deberán conocer con claridad cual será el producto final de su trabajo con la webquest y cómo habrán de realizarlo: Generación de documentos, elaboración de reportajes, encuestas, trabajos de síntesis, resolución de problemas, superación de cuestionarios de evaluación...

3. Proceso.- Descripción detallada y operativa de los pasos (subprocesos) que seguirán los alumnos para completar la tarea. Por lo general, los elementos a incluir son: Lista de tareas y cronograma (secuenciación temporal del trabajo). En algunos casos el profesor puede incluir pequeñas pistas o documentos que clarifiquen el orden a seguir o relacionen determinadas partes del proceso con algunos recursos.

4. Recursos.- Diferentes materiales accesibles en Internet (sitios web, ficheros de sonido, gráficos, videoclips, pequeños programas...) que el alumnado deberá emplear en las tareas encomendadas. En algunos casos, también es conveniente incluir otros recursos TIC disponibles en el centro: Base de datos de la biblioteca, vídeos, materiales multimedia, software educativo, etc.

5. Evaluación.- Sección del protocolo donde se indican con claridad los aspectos en que va ser evaluado el trabajo del alumno, especificando los instrumentos y los criterios de evaluación. La mayor parte de las webquest suelen emplear una matriz de rúbrica como plantilla de evaluación.

6. Conclusión.- Se ofrece un espacio al alumnado para la reflexión, individual o en grupo, sobre lo aprendido en el proceso. La conclusión puede ser formal (elaboración de resúmenes, informes de resultados...) o informal (comentarios o frases de los alumnos en el libro de visitas, o mediante algún tipo de formulario interactivo, en webquests más elaboradas.)

7. Guía didáctica.- Manual de uso y aspectos técnicos de la webquest. Dirigido, principalmente, a la orientación didáctica a otros profesores que quieran emplear este protocolo con sus alumnos. La filosofía de la webquest -al menos originariamente- sigue las reglas del freeware y la norma GPL. Por este motivo, hay numerosos portales educativos que recopilan webquests organizadas por materias y niveles educativos.

\section{Bases teóricas del protocolo didáctico.}

Cualquier propuesta didáctica empleando las TIC requiere, al menos como petición de principio, una referencia expresa a las bases filosóficas del modelo pedagógico en que se sustenta. Desde un punto de vista gnoseológico puede afirmarse, en conformidad con el principio de verifibilidad de Popper, que las teorías son redes conceptuales diseñadas para atrapar eventos del "Mundo1". Así pues, toda concreción didáctica establecida sobre la base de un "corpus teórico" de enunciados psicopedagógicos deberá ser entendida como protocolo experimental concebido para verificar o falsar uno o varios de los elementos programáticos de dicha teoría. 
Las webquest se asientan en una pedagogía constructivista interesada por el desarrollo de procesos cognitivos facilitadores del aprendizaje significativo y de la adquisición de las habilidades instrumentales necesarias para que el alumnado adquiera cierta autonomía educativa.

Los presupuestos teóricos de este modelo pueden sintetizarse en las cuatro siguientes categorías generales:

1. Apropiación del aparato teórico de la teoría de las Inteligencias Múltiples (Gardner, 1993), que asigna un valor diferencial a las aptitudes concretas del alumnado susceptibles de estimulación en un entorno educativo adecuado. De este modo, se diseña el protocolo atendiendo a un contexto motivacional abierto en el que múltiples destrezas, capacidades e intereses concurren, de manera colaborativa, en la resolución de una tarea. En este sentido el diseño de actividades en una webquest deberá integrar, al menos de manera implícita, procesos de sensibilización (Inteligencia Emocional), de planificación, organización y elaboración (Inteligencia Analítica), de construcción del conocimiento y formulación de hipótesis (Inteligencia Creadora), de trabajo colaborativo (Inteligencia Social) y de aplicación (Inteligencia práctica).

2. Asunción parcial de la hipótesis del aprendizaje auto-regulado (Zimmerman y Schunk, 1989), según la cual los roles docentes y discentes experimentan en algún punto del proceso de enseñanza-aprendizaje una transición desde el hetero-control hasta el autoaprendizaje limitado. De este modo, el valor de aprender no consiste tanto en la transmisión y fijación de conocimientos, ni siquiera en la construcción de conocimientos nuevos a partir de unos elementos curriculares y didácticos establecidos "a priori", sino en el proceso en virtud de cual el alumno adquiere destrezas específicas (cognitivas y metacognitivas) para consolidar cierta autonomía educativa. Algunos elementos de las webquest satisfacen plenamente los cuatro procesos reguladores presentes en el modelo de Pintrich (2000): a) Planificación, b) autoobservación, c) control y d) evaluación. Otros, van mucho más allá y hacen un guiño a las sólidas bases socio-educativas en que se asienta el paradigma de Vygotsky (1978).

3. Numerosos estudios sobre la ZDP asignan al postulado de la mediación social un papel hegemónico en los procesos educativos. Sin embargo, olvidan -quizá deliberadamente- las raíces genuinamente marxistas del programa de Vygotskiano, que interpreta la evolución del individuo como un proceso dialéctico en virtud del cual todos los cambios madurativos en los patrones de actividad psíquica están irreductiblemente vinculados a la actividad practicoproductiva. De esto modo, cualquier instrumento mediacional empleado en tareas docentes solo se revelará efectivo cuando estimula la coordinación de acciones intersubjetivas, idealmente asentadas en una "red difusa" de actividades culturales, tramas de significación, marcadores de contexto, descriptores de acción, etc. que, mediante la interacción grupal, acabarán concretándose en estructuras conceptuales y operacionales interiorizadas por el sujeto.

El protocolo de Dodge está concebido para favorecer la puesta en común de los conocimientos adquiridos por los alumnos tanto en las etapas de búsqueda como en las de construcción y resolución, asignando, de este modo, a las actividades colaborativas -tanto virtuales como presenciales- un papel central en el diseño de la webquest.

4. Aprendizaje multicanal.- Las Webquest bien diseñadas saben sacar partido a los dos componentes más valiosos de Internet : Multimedia e hipertexto.

Las teorías sobre las ventajas del aprendizaje multicanal no son nuevas, se remontan a los estudios de Hartman [4] (1961), Severin [5] (1967) y Paivio [6] (1971). Más recientes son los trabajos de Moore, Burton y Myres (1996), que avalan las hipótesis multicanal, subrayando que los entornos multimedia incrementan de manera efectiva el aprendizaje. Jonassen y Reeves (1996), desde un enfoque no muy alejado del paradigma mediacional, investigaron el valor del hipertexto como elemento facilitador en la construcción significativa del conocimiento. En la misma línea, Kommers y Lanzing (1997) 
consideran que el hipertexto puede contribuir a facilitar el autoaprendizaje, favoreciendo la búsqueda significativa al permitir al alumno una navegación autónoma, en la que es posible controlar los procesos de selección informativa y de profundidad conceptual. La proliferación de hiperenlaces sitúa al internauta en un escenario holista, en el que la sensación de ubicuidad, la inmediatez y la ruptura de la linealidad discursiva se convierten en elementos dominantes de esta nueva forma de "aprender" (en el sentido Vygotskiano de apropiación por participación) y "aprehender" (quizá al estilo de Heiddeger, como modo de "estar-en-el-mundo.") Rouert y Jarmo (1996) consideran la interacción hipertextual más próxima a la estructura reticular de la mente y, en particular, al modo en que los conceptos son almacenados en la memoria.

Los defensores de este protocolo, al analizar el marco teórico en el que encuentran acomodo las webquest, citan un largo corolario de principios metodológicos, psicológicos y filosóficos derivados de las cuatro categorías generales ya señaladas. Me he permitido resumirlos en el siguiente decálogo:

I) Aprendizaje basado en proyectos. Como concreción del aprendizaje situado. Se centra en focalizar la actividad del alumno hacia propuestas didácticas encaminadas al desarrollo y la producción de conocimientos. El proceso estará guiado, de manera flexible, por los elementos que integran el protocolo:

a. La tarea, que deberá concretar los objetivos y especificar con claridad el resultado final del trabajo que realizarán los alumnos.

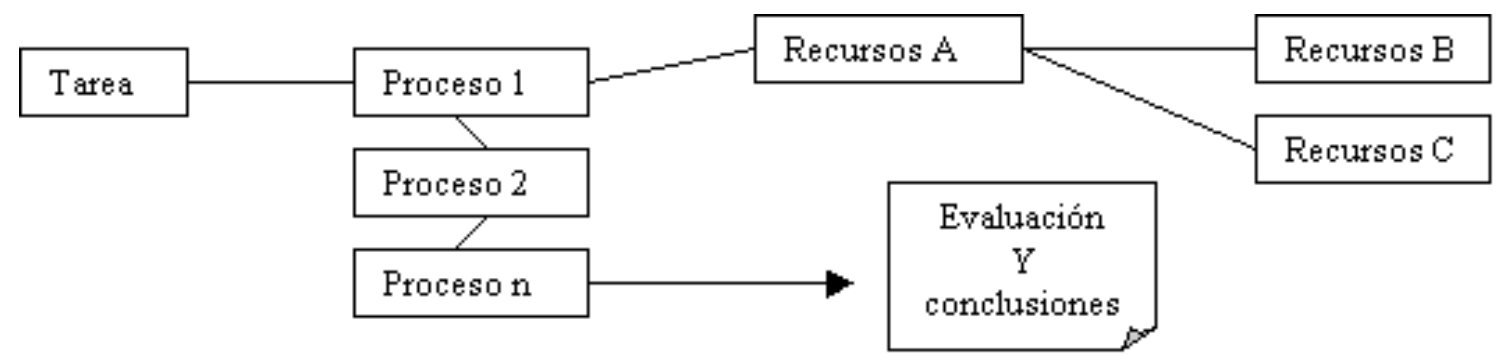

b. Los procesos; secuencia de pasos o actividades que deberán seguir los alumnos para completar la tarea, junto con los recursos necesarios para su consecución.

II) Capacidad del alumno para construir esquemas de conocimiento y estrategias eficientes de trabajo que le posibiliten asimilar experiencias significativas. En muchas webquest suele se frecuente el recurso a sistemas predefinidos de representación del conocimiento: Esquemas, diagramas de flujo, mapas conceptuales, etc. Por otro lado, las estrategias de trabajo están determinadas por la naturaleza de las tareas a realizar: Distribución grupal, asignación de roles, asunción de responsabilidades específicas, protocolos para el debate, puestas en común y presentación de resultados, etc.

III) Desarrollo del pensamiento crítico mediante la elaboración de actividades utilizando la información recopilada para realizar análisis inductivos y deductivos, contrastación de hipótesis, razonamiento por analogía, planificación, formulación de juicios bien fundados y, en general, cualquier tarea que estimule el desarrollo de la inteligencia en los niveles de operación y resolución.

IV) Pensamiento creativo. Las técnicas de la "tormenta de ideas" pueden emplearse con cierta eficacia en la producción de conocimientos nuevos: La flexibilidad de las tareas, la originalidad en el planteamiento de los procesos y, en particular, cualquier estrategia que favorezca la libre expresión de pensamientos novedosos son buenas herramientas para estimular la creatividad en el alumnado.

V) Questioning.- Es asombroso el poder de mayéutica en entornos colaborativos y su capacidad para favorecer el acercamiento a los problemas. El viejo "arte de hacer preguntas" vuelve a ser recuperado como ejercicio de reflexión sistemática -y no pocas veces de catarsis colectiva- Este proceso retórico suele estar dirigido por una serie de cuestiones clave (key questions) que el docente irá desgranando a medida que avanza el diálogo. Cuestiones elaboradas no con la intención de aproximar al alumno a la 
"verdad" sino de provocar disonancias cognitivas; suscitar dudas, erosionar prejuicios, someter a examen crítico ideas preconcebidas, con el fin de que el interlocutor acabe llegando por si mismo a aquello que se pretende. Algunas tareas de las webquest como las de consenso, las basadas en el misterio o las de autoconocimiento son ideales para la aplicación del método socrático.

VI) Autenticidad.- "Es más importante el cómo se enseña que el qué se enseña". Este tópico de la filosofía constructivista es elevado a la categoría de axioma por los defensores de las webquest.

VII) Estimulación de las diferencias individuales. El profesor deberá establecer para cada tarea general un diseño de actividades y procesos que sea compatible con la diversidad cognitiva (inteligencias múltiples) y con los estilos de aprendizaje del alumnado. En propuestas didácticas más elaboradas, se pueden especificar recorridos múltiples en la resolución de tareas.

VIII) Ruptura de la linealidad discursiva. La profusión de hipervínculos es una característica inherente a la estructura de cualquier documento web. Lo que favorece el desarrollo de estrategias divergentes en los procesos de búsqueda y selección de la información especificados para completar una tarea. En este sentido, los procesos y actividades diseñados por el docente para la realización de un protocolo didáctico "orientan" pero no "determinan" la actividad del alumno. El recorrido está guiado, en lo esencial, hacia la consecución de unos objetivos curriculares, pero los caminos son múltiples y las bifurcaciones impredecibles.

IX) Superación de la textualidad, haciendo uso intensivo de la multiplicidad y riqueza de formatos multimedia que ofrece la Red. Una webquest no es un entorno virtual, sino un protocolo diseñado para guiar los procesos de enseñanza-aprendizaje aprovechando el virtualmente infinito caudal de recursos disponibles en Internet. Al profesor corresponde establecer los elementos multimedia más apropiados para cada tipo de tarea.

\begin{tabular}{|l|l|}
\hline & Textos (HTLM, DOC, PDF...) \\
& Bases de datos documentales \\
& Bancos de imágenes \\
& Ficheros de audio \\
& Videoclips \\
\hline & Videoclips \\
\hline Tareas de misterio & Juegos de rol \\
& Mundos virtuales \\
& Videojuegos \\
& MUD \\
\hline Tareas creativas & Visitas virtuales a museos \\
& Software de desarrollo de la creatividad \\
\hline Tareas científicas & Juegos en red \\
& Programas de diseño \\
\hline & Sitios web dedicados a la ciencia. \\
& Laboratorios virtuales \\
& Software específico \\
\hline & Hojas de cálculo \\
\hline & Foros. \\
\hline
\end{tabular}




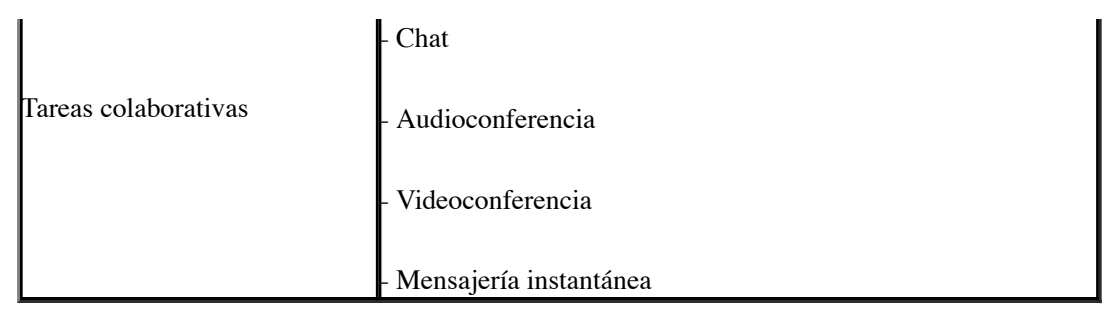

X) Interactividad y aprendizaje colaborativo. La mediación interpersonal e instrumental es un factor determinante del modelo. Muchos de los procesos requieren la asunción de roles y la interacción presencial entre grupos de alumnos, otros tienen lugar en escenarios virtuales empleando herramientas como foros, chat, videoconferencia, etc. A día de hoy, no existe ningún estudio que avale ventaja alguna de unos frente a otros. El profesor, en función de los objetivos buscados, elegirá el que mejor se adapte a cada tipo de tarea.

\section{Tipología de webquests: tareas, procesos y recursos.}

Existen dos variedades de webquest: Short Term Webquests [7] , que desarrollan aspectos puntuales de una unidad didáctica, y cuya duración no suele ser superior a dos sesiones de 50 minutos; y Longer Term Webquests, diseñadas para el estudio en profundidad de uno o varios temas establecidos en la programación de una determinada área de conocimiento o asignatura. La realización de las actividades contenidas en el protocolo puede ser lineal o alternarse con otras prácticas docentes. Su duración típica oscila entre las cinco y quince sesiones.

El primer tipo de Webqest se centra en una única tarea, articulada en un repertorio de procesos, por lo general, definido y cerrado. El segundo, por el contrario, tiene por objeto el análisis, producción, adquisición e integración de un cuerpo amplio de conocimientos y/o destrezas, pudiendo combinar varias tareas organizadas en torno a un conjunto más flexible de procesos y recursos.

Por otro lado, las tareas encomendadas a los alumnos determinan la metodología de trabajo empleada en diferentes situaciones de enseñanza-aprendizaje y los recursos necesarios para el desarrollo de procesos y subprocesos. No resulta sencillo establecer una clasificación de tareas. En general -y de manera aproximada- seguiré en el presente estudio la taxonomía inicialmente propuesta por Dodge [8]

\section{Tareas de repetición.}

Actividades basadas en la búsqueda, asimilación y comprensión de determinados contenidos informativos previamente establecidos por el docente. Se trata, como reconoce el propio autor de las webquest, de las tareas más próximas a la enseñanza tradicional y menos innovadoras, pero siguen mostrándose efectivas en numerosas prácticas escolares.

El formato suele ser breve, a menudo de una única sesión [9] que se resuelve en unos pocos pasos bien definidos. Su estructura sigue un esquema similar a este:

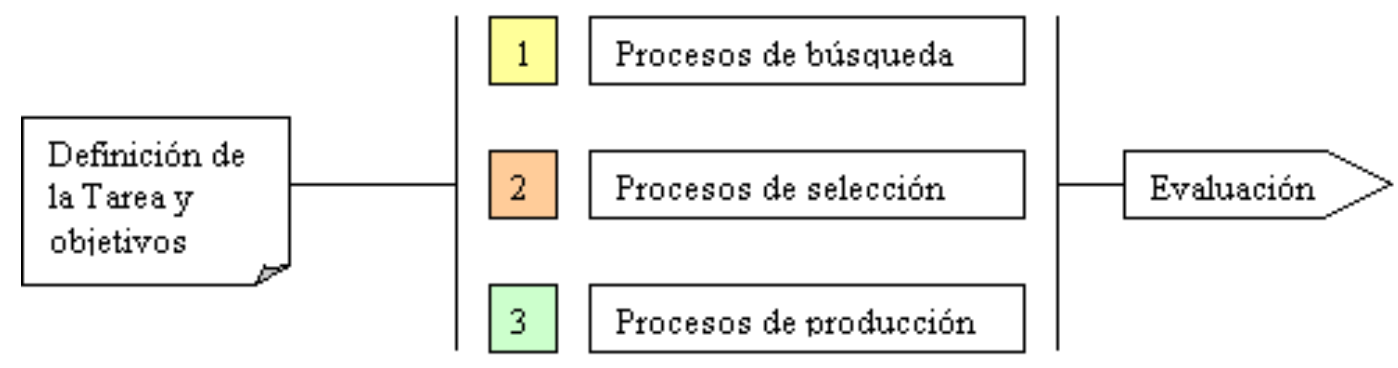

Los procesos de búsqueda se centran, por lo general, en un pequeño listado de recursos web idealmente multiformato- que el alumno deberá visitar en un orden prefijado. 
La selección de información no deberá ser repetitiva, ni limitarse al consabido "cortar y pegar". Es muy recomendable que el profesor tenga previsto algún tipo de plantilla de recogida de datos que obligue al alumno, aún de manera elemental, a filtrar, comparar y resumir las diversas fuentes.

La producción de un informe elaborado con el procesador de textos, de una pequeña presentación multimedia o de un simple esquema con el que participar porteriormente en algún debate, suelen ser muy habituales en este tipo de tareas.

\section{Tareas de recopilación.}

Centran su interés en la búsqueda de información multimedia sobre un determinado tema, poniéndola en un formato común o procesándola de algún modo. La elaboración de galerías de imágenes, determinados ejercicios de fotocomposición o el desarrollo de auténticos museos virtuales constituyen webquests que entran dentro de esta categoría.

Los procesos implicados deberán centrarse en la selección, transformación y producción de contenidos novedosos. El alumno deberá sentir que participa en un proceso creativo en el que la búsqueda de información representa solo el primer paso. Los materiales generados deberán publicarse en la Red u organizarse en presentaciones, boletines o pequeñas revistas digitales.

\section{Tareas de misterio.}

La asunción de personalidades imaginarias y algún juego de rol sencillo pueden ser buena base para construir una webquest que motive al alumnado. Se suele recomendar este tipo de actividades didácticas para alumnos de los primeros ciclos de Primaria. Sin embargo, esta metodología resulta fácilmente adaptable a los niveles de Secundaria y Bachillerato, siempre y cuando se especifiquen con claridad los objetivos curriculares que se pretenden conseguir, los contenidos o destrezas sobre los que se va a trabajar y, naturalmente, no se trivialice demasiado con juegos inapropiados para las edades a las que va dirigido el protocolo.

Existen otras propuestas como las "búsquedas de tesoros" o las "aventuras conversacionales" que no constituyen una webquest, ya que el objetivo de estas últimas está encaminado a la producción de contenidos de algún tipo por parte del alumnado.

\section{Tareas periodísticas.}

Híbrido entre las tareas de rol y las de recopilación de información. Se pide a los alumnos que actúen como reporteros de una agencia de noticias, buscando pistas sobre un hecho, situación o personaje. La actividad implica contrastar fuentes diversas, calibrando su exactitud y veracidad, recopilar datos en múltiples soportes, organizar la información disponible, filtrarla, realizar análisis valorativos y elaborar un informe con estilo periodístico que posteriormente podrá ser publicado en Internet o formar parte de la revista del centro.

Este tipo de tarea -más compleja que las anteriores- requerirá un mayor número de sesiones, una planificación de procesos abierta y flexible, que potencie la iniciativa y la autonomía del alumno, así como la organización del grupo en varios equipos de trabajo.

Un posible esquema de este tipo de tarea sería: 


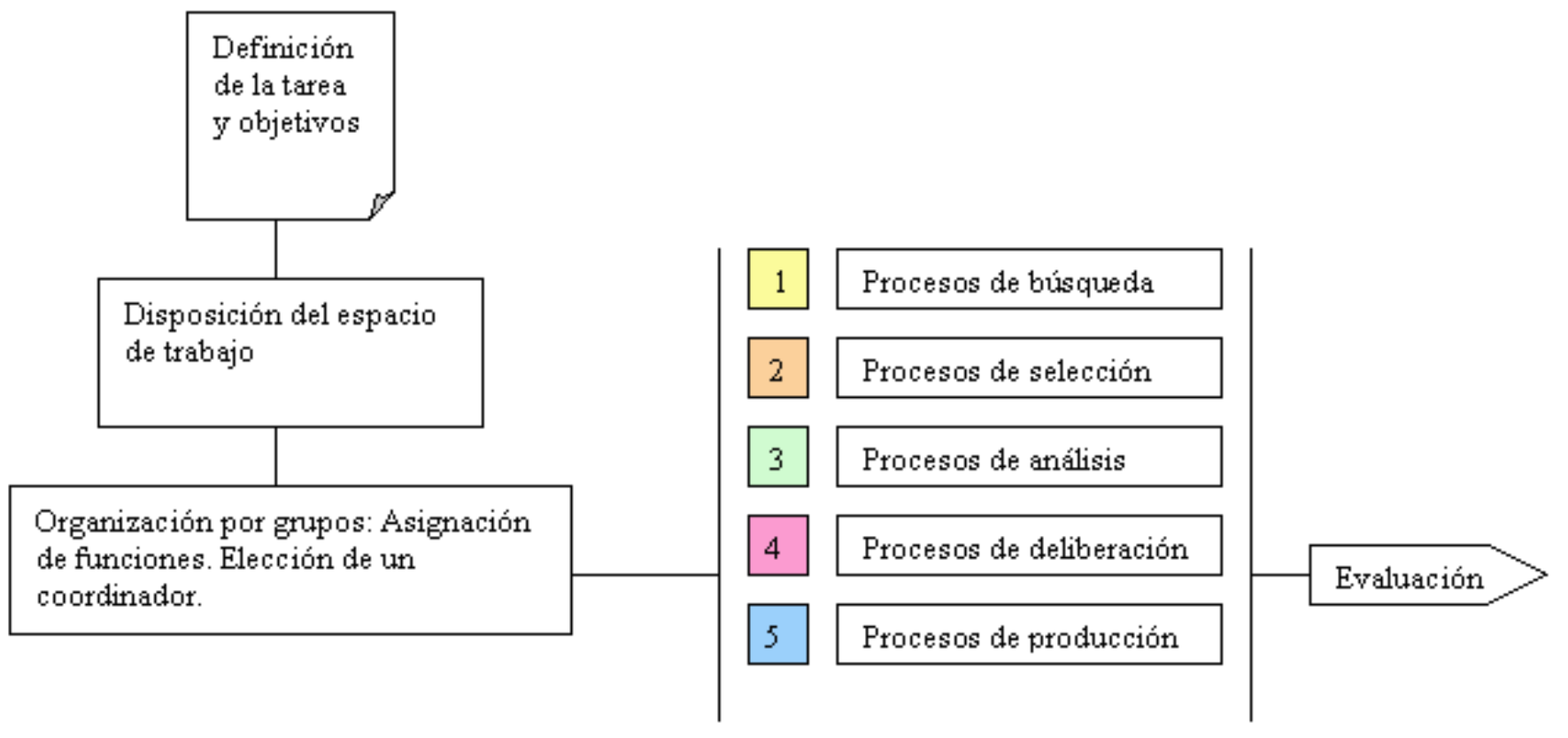

\section{Tareas de diseño.}

Se pide a los alumnos la creación de un protocolo o plan de acción con fines productivos, estratégicos $\mathrm{u}$ organizativos. La meta, por tanto, consiste en alcanzar un objetivo cumpliendo ciertas especificaciones establecidas de antemano. Por ejemplo, idear una ruta óptima para vender determinado producto estableciendo ciertas restricciones de tiempo y de consumo de energía, puede ser considerado como tarea de diseño logístico; mientras que diñar los pasos para la fabricación y ensamblaje de un pequeño electrodoméstico, sería una tarea de diseño productivo.

Una tarea de este tipo resulta apropiada para estimular la inventiva, las dotes organizativas, y el trabajo en equipo. Requiere una planificación muy detallada de la secuencia de procesos: (a) Descripción y documentación; (b) plan de trabajo, fases, recursos, materiales; (c) desarrollo, ejecución; (d) presentación del producto y valoración de resultados.

\section{Tareas de consenso.}

Tienen como objetivo la asunción puntos de vista divergentes sobre un mismo tema. Los procesos discursivos centrados en el debate, la argumentación racional y la búsqueda del consenso constituyen el eje de estas propuestas didácticas. El tema propuesto deberá ser estimulante y abierto, e incidir sobre cuestiones que realmente afecten a los alumnos y por las que éstos sienta de antemano cierto interés. Los escenarios para el debate pueden ser presenciales o virtuales. Empleándose, en este último caso, herramientas colaborativas específicas como foros temáticos, chat, pizarra electrónica o videoconferencia.

El siguiente cronograma muestra un esquema general de trabajo para este tipo de tarea:

\begin{tabular}{|c|c|c|}
\hline 1 & $1^{\mathrm{a}}$ SESIÓN: & Descripción de la actividad. \\
\hline 2 & & Distribución del espacio de trabajo. \\
\hline 3 & $50 \mathrm{~min}$. & Organización grupal de alumnos. \\
\hline 4 & & Procesos de búsqueda de información. \\
\hline 5 & 2a SESIÓN: & Selección, elaboración y síntesis. \\
\hline 6 & & Asunción de puntos de vista. \\
\hline 7 & $50 \mathrm{~min}$. & Deliberación por grupos. \\
\hline 8 & 3 $^{\text {a }}$ SESIÓN: & Nombramiento de un portavoz por grupo. \\
\hline 9 & & Exposición argumentativa. \\
\hline 10 & $50 \mathrm{~min}$. & Debate intergrupal. \\
\hline
\end{tabular}




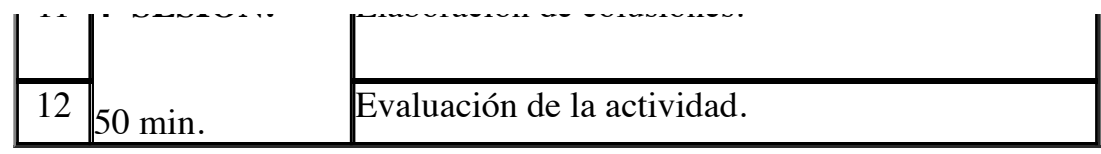

\section{Tareas de persuasión.}

Que persiguen capacitar los alumnos para el empleo de técnicas específicas de la lógica informal y el diálogo argumentativo. Se trata de una variante de la retórica en la que dos o más participantes intercambian puntos de vista observando ciertas reglas propias la argumentación racional. El objetivo no es tanto convencer a la audiencia, sino colaborar de forma crítica en el análisis de un tema mediante la confrontación de argumentos, creencias y convicciones. Un caso recurrente en este tipo de tare es el "formato judicial". Temas de fuerte contenido ético o social, como la pena de muerte, la eutanasia, la sociedad de consumo, las guerras, el racismo, la globalización, la manipulación informativa, etc., resultan especialmente apropiados para esta modalidad de webquest.

\section{Tareas de autoconocimiento.}

En labores de tutoría, suele resultar obligado el empleo de técnicas para reforzar la autoestima de los alumnos y hacer que se integren en el grupo, mediante el conocimiento mutuo y respeto a la diversidad. Una tarea de este tipo bien planteada deberá forzar a los alumnos a compartir de manera desinhibida cuestiones personales con el resto de sus compañeros, reforzando los vínculos interpersonales -y resolviendo en no pocas ocasiones posibles conflictos- a través del conocimiento mutuo, el diálogo sincero y la apertura a los demás.

Otra variante más formal, consiste en emplear estas webquest en actividades de orientación escolar, como la toma de decisiones sobre cuestiones relativas al estudio, la elección de una carrera o el futuro laboral de los alumnos.

\section{Tareas creativas.}

Persiguen estimular las dotes artísticas e inventivas del alumno a partir de una serie de materiales multimedia disponibles en la Red. Los alumnos podrán elaborar un producto literario, musical, fotográfico, etc. en respuesta a los objetivos de aprendizaje establecidos en la webquest. En este caso, tanto los procesos como la secuenciación temporal y los criterios de evaluación serán necesariamente más flexibles y abiertos.

\section{Tareas analíticas.}

Comparar, analizar secuencias de sucesos, establecer relaciones causales entre fenómenos e incluso formular hipótesis explicativas sobre determinados aspectos de la realidad son tareas didácticas claramente presentes en la filosofía constructivista. En este tipo de actividades no se valora tanto el producto final, como los procesos de razonamiento y las técnicas de representación de la información empleadas. Conviene que las tareas encomendadas obliguen al alumnado a considerar los problemas desde múltiples perspectivas, a especular sobre el significado profundo de las relaciones encontradas y a desplegar sus propias conclusiones y estrategias lógicas en marcos conceptuales más amplios.

Una webquest de este tipo podría responder al siguiente esquema: 


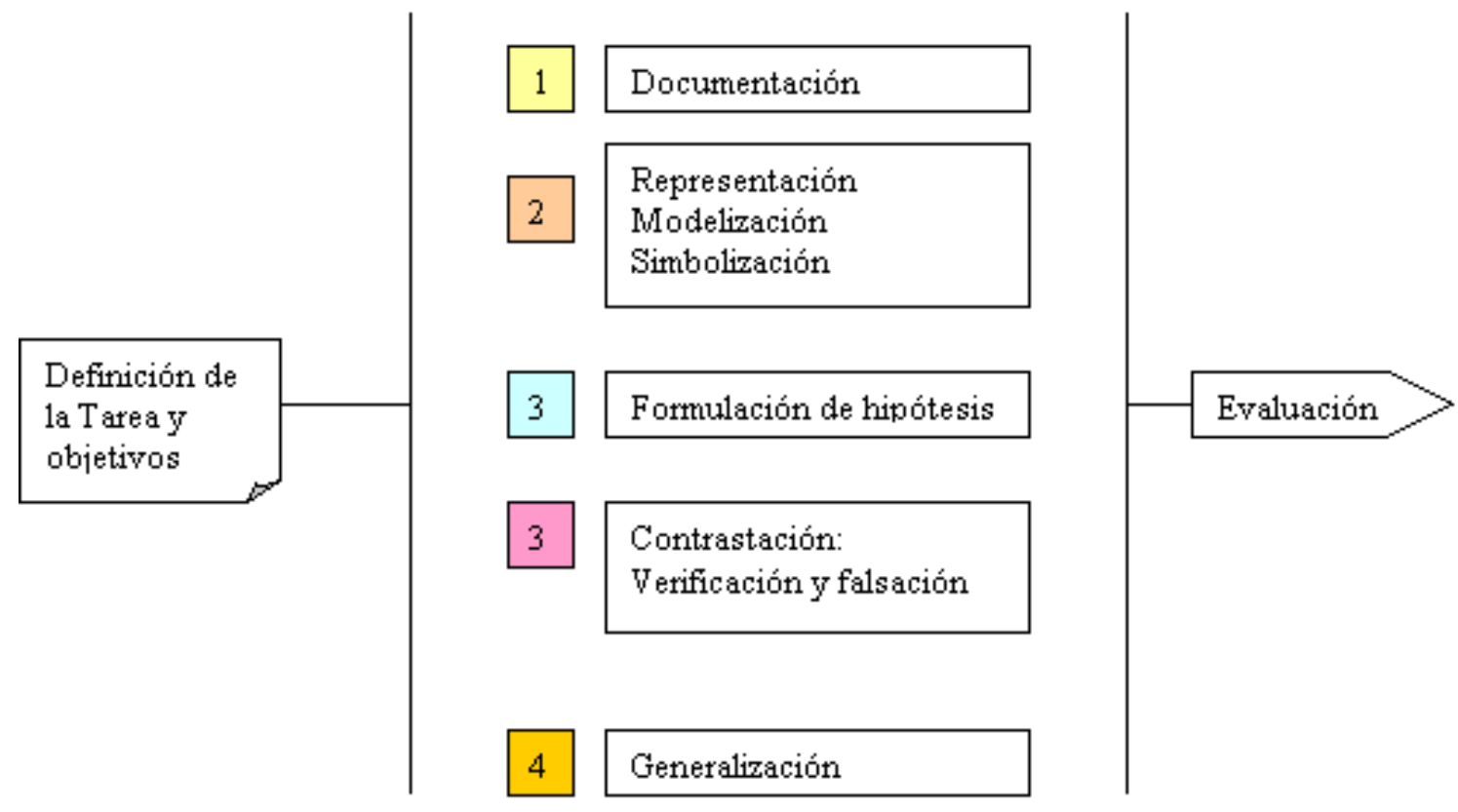

\section{Tareas de formulación de un juicio.}

Obligan al alumno a tomar una decisión a partir de una serie de pruebas o argumentos bien fundados. El resultado toma la forma de una exposición razonada, o "informe de caso" en el que se elige una entre varias alternativas, pudiendo debatirse luego el resultado de la elección con los demás compañeros. En determinadas situaciones, será conveniente que el protocolo especifique las alternativas posibles, consistiendo la tarea del alumno en la recopilación de pruebas que le permitan escoger, apoyándose en argumentos empíricos y racionales, alguna de ellas. Otro tipo de actividades, también exigirán al alumno una propuesta razonada de las alternativas posibles en la resolución de un problema.

\section{Tareas científicas.}

Estas tareas persiguen familiarizar al alumno con los diferentes procedimientos de investigación científica. Las actividades contenidas en el protocolo deberán acomodarse a las características específicas de cada método. Por ejemplo, en las Ciencias Empíricas, el alumno deberá realizar tareas en las que se ejerciten los pasos del método hipotético-deductivo, poniendo especial énfasis en los aspectos relativos a la construcción de enunciados protocolarios, formulación de hipótesis y el desarrollo de situaciones experimentales que permitan su verificación o falsación. En las Ciencias Sociales, se atenderá a las técnicas cuantitativas y cualitativas de recogida de información y al tratamiento estadístico de la misma, procediendo luego a la construcción de modelos explicativos que satisfagan una serie de requisitos previamente establecidos por el docente. Este tipo de wequest resulta especialmente indicada para alumnos de Bachillerato.

Por lo que se refiere las habilidades mentales o procesos de pensamiento que tratan de estimular las distintas clases de webquest, Dodge [10] , dando por buenos los estudios de Marzano (1992), se limita a señalar los siguientes:

1. Comparar: Identificación y análisis de las similitudes y diferencias entre objetos y conceptos.

2. Clasificar: Agrupar objetos o conceptos en categorías diferenciables en base a una serie de atributos.

3. Inducir: Capacidad de inferir generalizaciones a partir de la observación y el análisis de casos, fenómenos, situaciones o premisas particulares.

4. Deducir: Extraer consecuencias y principios particulares a partir de leyes y teorías generales. 
5. Análisis de errores: Identificación de errores lógicos, conceptuales o procedimentales en nuestro propio razonamiento o en el de otros.

6. Construcción de pruebas (sistematización): Construcción de un marco conceptual que sirva de soporte y ofrezca respaldo a nuestras convicciones.

7. Abstracción: Identificar el tema o estructura subyacente en cualquier elemento discursivo o procedimental.

8. Análisis en perspectiva (contextualización): Identificar y analizar las diferentes perspectivas sobre una determinada cuestión.

Considero necesario añadir a estas ocho habilidades descritas al menos otras dos que hagan referencia a los procesos colaborativos y de interacción social que se desarrollan en numerosas webquest:

9. Deliberación, negociación: Capacidad o habilidad discursiva propia del debate y del intercambio de ideas.

10. Argumentación: Capacidad de expresar nuestras ideas y convicciones, defendiendo nuestros puntos de vista mediante el diálogo argumentativo.

Concluiré este estudio sobre el valor pedagógico de las webquest elaborando una tabla, a modo de conclusión, en la que trato de relacionar los tipos de tareas con los procesos, las herramientas TIC y los productos elaborados por los alumnos. Dejo pendiente para ulteriores trabajos sobre el tema, las técnicas de recogida y representación de la información, los estilos de aprendizaje implicados, los roles de docentes y discentes y la evaluación del protocolo.

\begin{tabular}{|c|c|c|c|}
\hline TAREAS & PROCESOS & HERAMIENTAS TIC & PRODUCTOS \\
\hline Repetición & \begin{tabular}{|l} 
Comparar \\
Clasificar \\
Análisis de errores
\end{tabular} & \begin{tabular}{|l} 
Buscadores \\
Bases de datos \\
Procesadores de texto
\end{tabular} & $\begin{array}{l}\text { Tablas } \\
\text { Resúmenes } \\
\text { Pequeños informes } \\
\text { Cuestionarios de evaluación }\end{array}$ \\
\hline Recopilación & $\begin{array}{l}\text { Comparar } \\
\text { Clasificar } \\
\text { Análisis de errores }\end{array}$ & \begin{tabular}{|l} 
Buscadores \\
Bases de datos \\
Soportes multimedia \\
Archivos \\
Bibliotecas
\end{tabular} & $\begin{array}{l}\text { Fichas catalográficas } \\
\text { Recensiones } \\
\text { Resúmenes } \\
\text { Pablas comparativas } \\
\text { Presentaciones }\end{array}$ \\
\hline Misterio & $\begin{array}{l}\text { Comparar } \\
\text { Clasificar } \\
\text { Deducir } \\
\text { Análisis en perspectiva }\end{array}$ & $\begin{array}{l}\text { Videojuegos } \\
\text { Aventuras conversacionales y juegos } \\
\text { de rol } \\
\text { Buscadores } \\
\text { Videoclips }\end{array}$ & $\begin{array}{l}\text { Puestas en común } \\
\text { Interacción en juegos de Rol } \\
\text { Resolución de adivinanzas y acertijos } \\
\text { Elaboración de relatos o pequeños } \\
\text { guiones cinematográficos } \\
\text { Elaboración de crucigramas, jeroglíficos, } \\
\text { sopas de letras } \\
\text { Dramatizaciones }\end{array}$ \\
\hline Periodísticas & $\begin{array}{l}\text { Comparar } \\
\text { Clasificar } \\
\text { Análisis de errores }\end{array}$ & \begin{tabular}{|l} 
Buscadores \\
Foros \\
Chats
\end{tabular} & $\begin{array}{l}\text { Elaboración de informes } \\
\text { Creación de artículos periodísticos } \\
\text { Presentaciones en formato multimedia }\end{array}$ \\
\hline
\end{tabular}




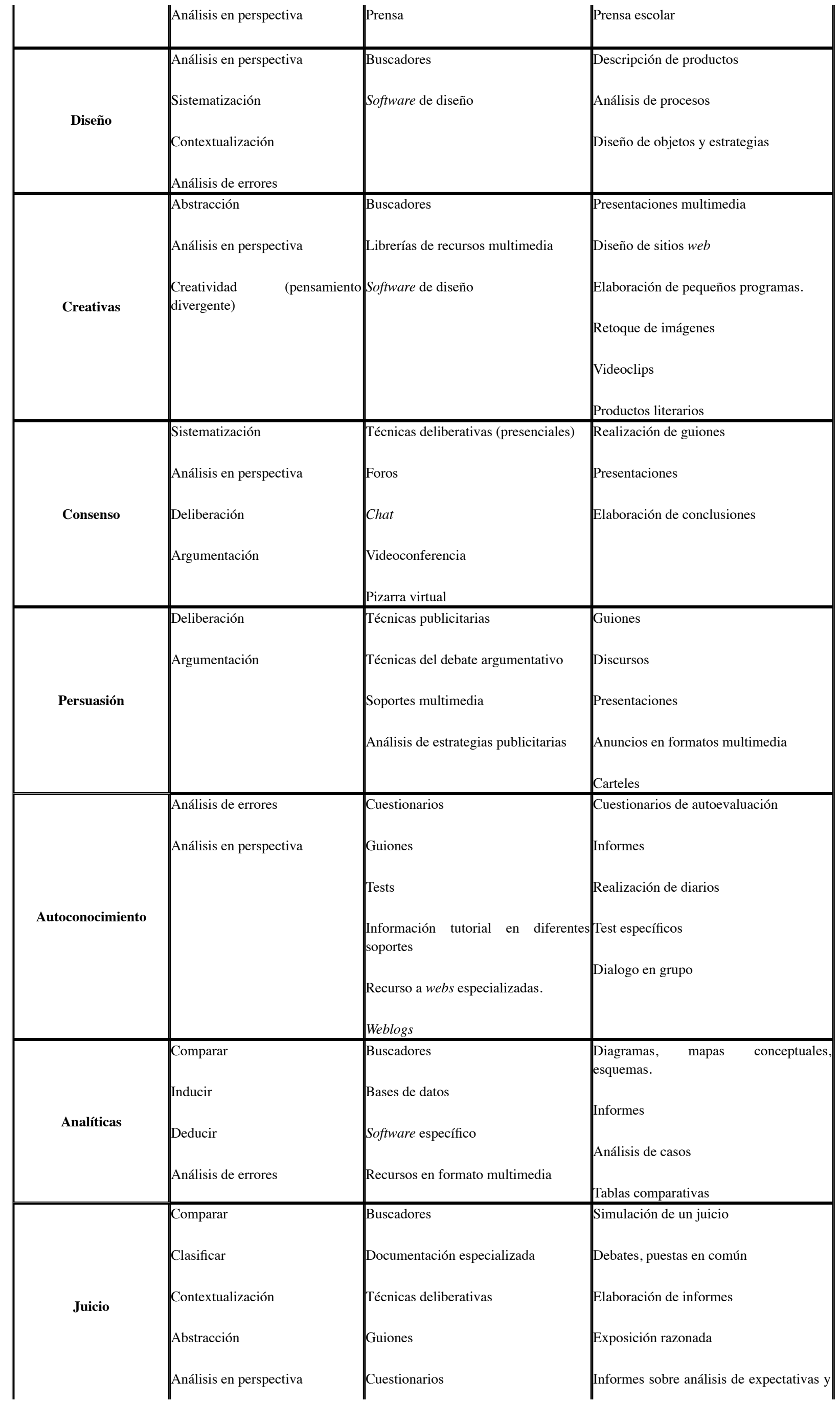




\begin{tabular}{|c|c|c|c|}
\hline & & Entrevistas & toma de decisiones \\
\hline Científicas & $\begin{array}{l}\text { Inducir } \\
\text { Deducir } \\
\text { Análisis de errores } \\
\text { Abstracción } \\
\text { Contextualización }\end{array}$ & $\begin{array}{l}\text { Buscadores } \\
\text { Recurso a webs especializadas } \\
\text { Saboratorio virtual } \\
\text { Software científico } \\
\text { Programas de simulación }\end{array}$ & $\begin{array}{l}\text { Elaboración de hipótesis } \\
\text { Informes científicos } \\
\text { Presentaciones multimedia } \\
\text { Pequeños modelos y simulaciones } \\
\text { Realización de tareas con hojas de } \\
\text { cálculo y programas de análisis } \\
\text { estadístico }\end{array}$ \\
\hline
\end{tabular}

\section{Referencias}

ADELL, J. (2004): Internet en el aula: Las webquest, Edutec. Revista de Tecnología Educativa, 17 http://www.xtec.es/ cbarba1/Articles/AdellWQ.pdf

ALONSO, M. C. y DOMINGO, J. G. (2000): Aprendizaje y ordenador, Dykinson, Madrid.

BARBA, C. (2002): La investigación en Internet con las Webquest, Comunicación y Pedagogía, 185, pp.62-66 http://www.cibereduca.com/webquest/carme-barba.pdf

BLANCO, S., y otros (2000): "Estudio de caso: Uso de las Webquest en Educación Secundaria" http://www.webquestcat.org/experien/estudio\%20caso\%20WQ\%20secundaria.PDF

BROOKHART, S. M. (1999): The art and Science of Classroom Assessment: The Missing Part of Pedagogy, ERIC Clearinghouse on Higher Education Washington DC.I BBB32577, George Washington Univ. Washington DC. (Una Buena recensión de la obra en: http://www.ericdigests.org/2000-2/art.htm

CARRIE, S. (2001): "The Effects of Visual and Verbal Cues in Multimedia Instruction" (Documento on-line) http://scholar.lib.vt.edu/theses/available/etd-03092001-

134749/unrestricted/DissertationITCBSREV3.pdf

DODGE, B. (1995): "Some Thougthts About Webquest" (Documento on-line) http://edweb.sdsu.edu/courses/edtec596/about_webquests.html

DODGE, B. (1999): "Webquest Taskonomy: A Taxonomy of Tasks" (Documento on-line) http://edweb.sdsu.edu/webquest/taskonomy.html

DODGE, B. (2001): "FOCUS: Five Rules for Writing a Great Webquest", Learning \& Leading with Technology, 28 (8): 6-9, 58. http://www.webquest.futuro.usp.br/artigos/textos_outros-bernie1.html

DODGE, B. (2004): "WebQuest design patterns. (Documento on-line) http://webquest.sdsu.edu/designpatterns/all.htm

DUDENEY, G. (2003): The Quest for Practical Web Usage, TESL-EJ v6 (4) http://wwwwriting.berkeley.edu/TESL-EJ/ej24/int.html

Gardner, H. (1993): Inteligencias Múltiples. La teoría en la práctica, Piados, Barcelona.

HARTMAN, F. R. (1961): Investigation of recognition learning under multiple-channel presentation and testing conditions, AV Communication Review, 9, pp. 24-43.

HARTMAN, F. R. (1961): Single and multiple channel communication: A review of research and a proposed model, AV Communication Review, 9, pp. 235-262. 
JONASSEN, D. y REEVES, T. (1996) "Learning with Technology: Using Computers as Cognitive Tools", Handbook of research for educational communications and technology (pp. 693-719). Macmillan, New York

KELLY, R. (2000): Working with WebQests: Making the Web Accessible to Students with Disabilities, Teaching Exceptional Children, 32 (6) 4-13 http://www.dldcec.org/pdf/teaching_howtos/working_with_webquests.pdf

KOMMERS, P. y LANZING, J. (1997): Student's Concept Mapping for Hypermedia: Navigation Through the WWW Space and Self-Assessment, Journal of Interactive Learning Research, (8), pp. 421-455.

MARTÍN, J. M, BELTRÁN, J.A. y PÉREZ, S.L. (2003): Cómo aprender con Internet, Foro Pedagógico de Internet. Fundación Encuentro, Madrid.

MARZANO, R. J. (1992):A different kind of classroom: Teaching with dimensions of learning. Alexandria VA: Association for Supervision and Curriculum Development.

MARZANO, R. J. y otros (1992):Dimensions of Learning Teachers' Manual. Alexandria, VA: Association for Supervision and Curriculum Development.

MOORE, D.M., BURTON, J. K. y MYERS R. J. (1996): Multiple-Channel Communication: The Theorical and Research Foundation of Multimedia, en D. H. JONASSEN (Ed.), Handbook of Research for educational communications and technology, pp.1213-1245. Simon \& Schuster Macmillan, New York.

NITKO, A. J. (1996): Educational assessment of students Englewood Cliffs, NJ: Merrill.

PAIVIO, A. (1971): Imagery and Verbal Processes, Holt, Rinehart \& Winston, New York

PINTRICH, P. R. (2000): The role of goal orientation in self-regulated learning. En BOEKAERTS, Handbook of self-regulation, Academic Press, San Diego (451-502).

PRADEEP, R. y otros (2004): Webquest in Social Studies Education, Journal of Interactive OnLine Learning, (v.3) 2. http://www.ncolr.org/jiol/archives/2004/fall/05/Index.pdf

REIGELUTH, Ch. (1999): Instructional Design Theories and Models: A New Paradigm of Instructional Theory, Mahwah, New Jersey.

ROUERT, J. F. y JARMO, L. (1996): Studying and Learning with Hipertext: Empirical Studies and their Implications, Hypertext and Cognition.

SAILER, G. y GRANT, G. (2001): "Piaget Webquest" http://pt3.nl.edu/saleredgingtonwebquest.pdf

SEVERIN, W.J. (1967): Another look at cue summation, AV Communication Review, 15 (4) pp. 233 245.

STARR, L. (2000): Meet Bernie Dodge -The Frank Lloid Wright of Learning Envirionments!, Education World (Revista on-line) http://www.educationworld.com/a_tech/tech/tech020.shtml

STERNBERG, R. J. (1997): Thinking Styles, The Press Sindicate of University of Cambridge

TIFFIN, J. y RAJASINGHAN, L. (1997): Hacia la clase virtual. La educación en la sociedad de la información, Paidos, Barcelona.

TILESTON, D.W. (2000): Ten Best Teaching Practices: How Brain Research, Learning Styles, and 
Standards Define Teaching. Thousand Oaks, Corwin Press, California.

VYGOTSKY, L. S. (1978): Mind in Society, Harvard University Press, Cambridge

WALVOORD, B. E., y ANDERSON, V. J. (1998): Effective grading: A tool for learning and assessment, Jossey-Bass, San Francisco.

WILSON, B. G. (1995): Metaphors for instruction: Why we talk about learning environments, Educational Technology, 35 (5), 25-30. http://carbon.cudenver.edu/ bwilson/metaphor.html

WILSON, B. G. (1996): Constructivist Learning Environments. Case Studies, Instructional Designs, Englewood Cliffs: Educational Technology Publications.

ZIMMERMAN B. J. y SCHUNK, D.H. (1989): Self-regulated learning and academic achievement: Theory, research and practice, Springer-Verlag, New York.

ZIMMERMAN, B. J. (1986): Development of self-regulated learning, Contemporary Educational Psychology, 81, pp. 329-339.

\section{Notas:}

[1] Profesor de Tecnología Educativa en la Universidad del estado de San Diego. Creador de las webquest, junto con Tom March en 1995. Su portal http://webquest.org/ se ha convertido en obligado lugar de referencia para los docentes interesados en la aplicación de esta Tecnología Educativa.

\section{[2] http://babylon.k12.ny.us/usconstitution/focus-5\%20rules.pdf}

[3] Existen normas específicas informalmente consensuadas. Todos los profesores que desarrollan webquest para su aplicación al aula y ulterior difusión en Internet asumen los apartados que vertebran el protocolo.

[4] Pionero en el estudio del potencial educativo de la televisión. Investigó el efecto que tienen los estímulos sensoriales presentados de manera simultanea en dos o más formatos diferentes, llegando a la conclusión (luego avalada por una constelación de estudios análogos) de que se produce un mejor aprendizaje cuando se muestra conjuntamente un texto asociado a imágenes fijas o en movimiento. Hoy día, su célebre modelo sobre las relaciones entre entradas sensoriales ("redundantes", "relacionadas", "no relacionadas" y "contradictorias"), sigue encontrando fácil acomodo en el marco de propuestas cognitivas más complejas.

[5] Autor del "Principio de adición de estímulos", según el cual la información multicanal solo es eficaz cuando se adicionan estímulos relevantes y no redundantes a través de dos o más canales sensoriales.

[6] Quien propuso la "Teoría del aprendizaje dual", analizando detalladamente los procesos mentales que permiten al sujeto el procesamiento simultaneo de dos fuentes de información: verbal (hablada o escrita) e icónica (imágenes fijas y en movimiento).

[7] En este apartado seguiré los términos empleados por Bernie Dodge en su artículo: "Some Thoughts About webquests", 1995. http://edweb.sdsu.edu/courses/edtec596/about_webquests.html

[8] Webquest Taxonomy: A Taxonomy of Tasks. http://edweb.sdsu.edu/webquest/taskonomy.html

[9] Lo que ha dado lugar a otra variedad del protocolo denominado Miniquest.

[10] "Some thoughts About Webquests". 
http://edweb.sdsu.edu/courses/edtec596/about_webquests.html

(C) Ediciones Universidad de Salamanca 